\title{
A one-trial response-choice technique for the biological study of memory '
}

BRYAN E. PFINGST AND RICHARD A. KING UNIVERSITY OF NORTH CAROLINA AT CHAPEL HILL

Rats given a $2 \mathrm{sec}, 2 \mathrm{~mA}$ foot shock for entering the right goal box of a T-maze showed a significant increase over a stable pre-shock baseline in choices of the left goal box on test trials given $24 \mathrm{~h}$ later. Such a one-trial learning task involving choice rather than latency as a measure of retention has certain advantages for studying the biology of memory.

If an animal is given an electroconvulsive shock (ECS) shortly after a task is learned, retrograde amnesia will result (Duncan, 1949). Since it is possible that multiple ECSs are aversive (Hudspeth, McGaugh, \& Thomson, 1964) or cause brain damage, it is desirable in studying the effects of ECS on memory to use a task which can be learned in one trial and therefore requires only one ECS to disrupt the memory. Of the several types of one-trial learning tasks available, one of the most popular for ECSmemory studies is the one-trial passive-avoidance task (e.g., Madsen \& McGaugh, 1961). In ECS studies using this task the $S$ is taught in a single trial to avoid making a response; the measure of retention used in such an experiment is the increase in latency of response. If an ECS is given shortly after the single learning trial, retrograde amnesia will result (McGaugh, 1966) and the $S$ will make the response he was taught to avoid making, i.e., his response latency will be low. The advantage of such a passive-avoidance task is that the low latency, interpreted as due to retrograde amnesia, cannot be interpreted as due to fear of ECS, while in an active avoidance task an interpretation involving fear of ECS is possible (see King, 1965 for further explanation).

ECS experiments involving passive avoidance have, however, recently been criticized. This criticism is based on the Idea that ECS may have a latencydecreasing effect independent of its amnesic effect. (See Chorover \& Schiller, 1966 for this argument.) The task to be presented in this paper retains the advantage of the one-trial passive-avoidance task but eliminates the objection raised by Chorover and Schiller by using choice rather than latency as an index of memory.

Method 2

The apparatus for this experiment was a $\mathrm{T}$-maze with a white start box and runway, gray arms and goal boxes, and a clear Plexiglas roof. In each goal box was a small white tray containing a Purina Lab Chow pellet. Throughout the experiment Ss could be reinforced for entering either goal box. Retracing was prevented by doors which closed behind $S$. Ss were allowed to eat for $20 \mathrm{sec}$ on each trial.
Ss were 18 adult male Wistar rats; they were maintained on a $23 \mathrm{~h}$ food deprivation schedule throughout the experiment. Prior to being run, Ss were handled daily for seven days and allowed to eat for 15 min each day in one of the goal boxes (alternate goal boxes from one day to the next). Then Ss were run 10 trials a day for seven days as follows: On Days 1-3 Ss were allowed free choices, except that no $S$ was allowed to enter the same goal box more than four times in a row. (Entrance could be prevented by closing a door.) On Days 4-7 all choices were free and a baseline was obtained for each $S$.

Since we intended to use as our measure of learning the increase in left choices after the learning experience, it seemed desirable at this point to eliminate any Ss whose baselines indicated a strong or increasing tendency toward left choices. Therefore any $S$ (1) choosing the left goal box more than eight out of 10 trials, or (2) increasing in the number of left choices two days in a row, was removed from the experiment. Three Ss were eliminated for these reasons.

On Day 8 the remaining 15 Ss were run as usual until they entered the right goal box. As soon as the right goal box was entered (this was often on the first trial) the food tray was pulled from the floor to the ceiling and a $2 \mathrm{~mA}$ scrambled foot shock was administered by a Grason-Stadler shock generator through the grid floor for 2 sec. The $S$ was then returned to its cage. On Day $9,24 \mathrm{~h}$ after the shock trial, the $S$ was given 10 free-choice trials and the number of right and left responses was measured. Results and Discussion

Table 1 shows the number of left goal box choices made by each $S$ on the four days before, and on the day after, shock in the right goal box. ${ }^{3}$ Note that 10 of the 15 Ss chose the left goal box on all 10 of the test trials; four of the remaining five showed an increase of three or more left choices over their pre-shock baselines. An analysis of variance showed that the change in behavior from Day 7 to Day 9 due to shock was highly significant $(F=89, \mathrm{df}=1 / 14$, $\mathrm{p}<$.001).

The pre-shock baselines were being taken on the same days that other rats (from this experiment and from other experiments not reported here) were being shocked and tested in the same maze. This controls for the possibility that rats being shocked in the right goal box might have left aversive cues (e.g., urine) in the goal box. Only two of the $18 \mathrm{Ss}$ showed an increase of more than two left choices 
Table 1 .

Number of left goal box choices made by each $S$ on the four days before and on the day after shock given in the right goal box

\begin{tabular}{|c|c|c|c|c|c|}
\hline \multirow[t]{2}{*}{$S$} & \multicolumn{4}{|c|}{ Baseline Days } & \multirow{2}{*}{$\begin{array}{r}\text { Test Day } \\
\text { Day 9* }\end{array}$} \\
\hline & Day 4 & Day 5 & Day 6 & Day 7 & \\
\hline$A-00$ & 1 & 1 & 0 & 1 & 8 \\
\hline A-11 & 1 & 2 & 0 & 0 & 10 \\
\hline A- 14 & 0 & 0 & 0 & 0 & 10 \\
\hline A- 15 & 1 & 1 & 1 & 1 & 8 \\
\hline A- 16 & 2 & 1 & 2 & 2 & 8 \\
\hline A- 17 & 3 & 0 & 1 & 2 & 3 \\
\hline A- 19 & 4 & 4 & 3 & 4 & 7 \\
\hline$A-22$ & 4 & 8 & 7 & 7 & 10 \\
\hline$A-23$ & 4 & 6 & 5 & 4 & 10 \\
\hline A. 24 & 2 & 4 & 1 & 2 & 10 \\
\hline A. 26 & 6 & 5 & 3 & 4 & 10 \\
\hline A- 28 & 2 & 1 & 0 & 0 & 10 \\
\hline A-33 & 2 & 3 & 1 & 1 & 10 \\
\hline A-34 & 0 & 0 & 2 & 2 & 10 \\
\hline A-37 & 0 & 1 & 0 & 0 & 10 \\
\hline Mean & 2.1 & 2.5 & 1.7 & 2.0 & 9.0 \\
\hline
\end{tabular}

* Foot shock given in right goal box on Day 8

over their baselines on the days that other rats were being shocked or tested in the maze.

We have demonstrated that this particular one-trial response-choice technique is workable; let us summarize its advantages for studying the effects of ECS and other amnesia-producing procedures on memory: (1) Because it is one-trial learning, only a single amnesia-producing procedure is required. (2) The change in behavior is produced by a single 2-sec stimulus; thus time-dependent processes (e.g., consolidation) can be accurately studied. (3) The retention test is one which involves choice; thus behavior interpreted as indicating amnesia cannot be attributed to non-memory effects of the amnesia-producing treatment on response latency. (4) It allows a design in which amnesia-producing procedures can be administered in such a way that behavior inter- preted as indicating amnesia cannot be interpreted as due to fear of the amnesia-producing procedure. (If amnesia is produced, the $\mathrm{S}$ will return to the goal box in which the supposedly fearful amnesia-producing procedure was administered.) (5) The task is remembered for at least $24 \mathrm{~h}$; thus adequate time for recovery from drastic amnesia-producing procedures, e.g., ECS, is available. (6) The results are reliable, with almost all Ss showing a marked change in behavior.

\section{References}

Chorover, S. L., \& Schiller, P. H. Reexamination of prolonged retrograde amnesia in one-trial learning. $J$. comp. physiol. Psychol., 1966, 61, 34-41.

Duncan, C. P. The retroactive effect of electroshock on learning. J. comp. physiol. Psychol., 1949, 42, 32-44.

Hudspeth, W. J., McGaugh, J. L., \& Thomson, C. W. Aversive and amnesic effects of electroconvulsive shock. J. comp. physiol. Psychol., 1964, 57, 61-64.

King, R. A. Consolidation of the neural trace in memory: Investigation with one-trial avoidance conditioning and ECS. J. comp. physiol. Psychol., 1965, 59, 283-284.

Madsen, M. C., \& McGaugh, J. L. The effect of ECS on one-trial avoidance learning. J. comp. physiol. Psychol., 1961, 54, 522523 .

McGaugh, J. L. Time-dependent processes in memory storage. Science, 1966, 153, $1351-1358$.

\section{Notes}

1. Supported by University of North Carolina Research Council Grant No. 324-ALU-1 (457).

2. Since this was a preliminary study for an ECS experiment, all Ss carried electrodes which were attached to jeweller's screws embedded in the skull. When the Ss were run in the maze, these electrodes were attached to an ECS apparatus by means of a wire which passed through a l-in. wide slit in the Plexiglas roof of

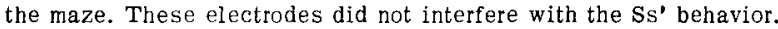
3. Latency plus running time, or total time, increased markedly over the pre-shock baseline on Trial 1 of the test day (Day 9); total time increased from a pre-shock average of $5.1 \mathrm{sec}$ to an average of $245 \mathrm{sec}$. This average does not include the slowest $\mathrm{S}$ who took $1 \mathrm{~h} 45 \mathrm{~min}$ to run the first test trial. (Some patience is required in using this technique.) 